\title{
Combining SVD-DCT Image Watermarking Scheme Based on Perona-Malik Diffusion
}

\author{
Sarra Belilita ${ }^{1}$, Nourredine Amardjia ${ }^{2}$, Tewfik Bekkouche ${ }^{3}$, Imen Nouioua ${ }^{1}$ \\ ${ }^{1}$ LCCNS Laboratory, Department of Electronics, Faculty of Technology, Ferhat Abbas University, \\ Setif, Algeria \\ ${ }^{2}$ LIS Laboratory, Department of Electronics, Faculty of Technology, Ferhat Abbas University, \\ Setif, Algeria \\ ${ }^{3}$ ETA Laboratory, Department of Electronics, Faculty of Technology, University of Bordj Bouarreridj, \\ Algeria \\ sara.belilita@univ-setif.dz
}

\begin{abstract}
In this paper, we propose an efficient image watermarking scheme based on combining Singular Value Decomposition (SVD) and Discrete Cosine Transform (DCT) transforms. The main idea consists of exploiting results obtained by the well-known Perona-Malik diffusion applied in image de-noising in watermarking domain by selecting significant blocks to embed a binary logo watermark. After applying the DCT on the selected blocks, the SVD is performed on each of these transformed blocks to modify a few numbers of its $\mathrm{U}$ matrix elements according to some defined conditions. Computer simulation and experimental results prove the efficiency of our proposed method against image processing attacks and outperform several previous schemes in terms of imperceptibility and robustness.
\end{abstract}

Index Terms-Digital image watermarking; 2D discrete cosine transform; Singular value decomposition; Perona-Malik diffusion.

\section{INTRODUCTION}

In recent years, the growth of the Internet and the development of powerful computing tools boosted up the digital multimedia e-commerce, which engendered an enormous presence on the web of digital multimedia data, such as audio, image, and video files. However, with the help of powerful multimedia processing tools, these files can be simply downloaded, easily modified, and then illegally distributed on the net. The protection of owners' intellectual property rights has become a major matter that is nowadays efficiently addressed by digital watermarking techniques. A digital watermarking technique consists of two procedures: an insertion procedure and an extraction procedure. By using secret keys, the insertion procedure embeds into the host multimedia content, usually called the cover, a watermark, which is a digital signature that holds the copyright information restricted solely to the cover's proprietor. By using the same secret keys, the extraction procedure permits to recover the watermark and consequently prove the ownership. An effective watermarking technique must provide some main aspects, which comprise [1]:

Manuscript received 6 January, 2019; accepted 10 April, 2019.
- A high imperceptibility: no apparent difference between the original and the watermarked contents should happen; - A realistic high robustness: the watermark should survive to some extent to intentional and unintentional attacks;

- A reasonable payload or capacity, which represents the amount of information contained in the watermark;

- A low complexity of the calculations, which determines the running time of the algorithm;

- A good reliability, which means that no other watermark than the built-in one is extracted. However, since some of these properties are antagonists, most of the watermarking techniques seek to have a reasonably good Imperceptibility-Robustness-Payload trade-off with a relatively low calculations' complexity.

Many watermarking techniques are developed especially at the still image level. According to the embedding domain of the cover, these techniques are set into either the spatial domain [2] or the transform domain [3]. Even if the spatial domain techniques are simpler to implement as they embed the watermark directly in the cover's pixels, the transform domain ones, which embed the watermark by slightly modifying the transform coefficients, provide higher robustness and better security requested in most watermarking applications. The transform methods can use one or a combination among several transforms like the discrete Fourier transform (DFT), the discrete cosine transform (DCT), the discrete wavelet transform (DWT), the singular value decomposition (SVD), etc. Transforms' combination techniques called hybrid techniques seek to achieve better robustness, superior imperceptibility, and higher security. Moreover, in relation to the watermark extraction procedure, digital watermarking schemes are categorized as non-blind, semi-blind, and blind. When processing the watermark detection, a non-blind watermarking scheme necessitates the original cover, the original watermark, and the secret keys. A semi-blind one needs only the original watermark and the secret keys. A blind scheme, which is undeniably the most challenging type, requires only the secret keys.

In recent years, many consistent SVD-based watermarking techniques have been considered [4]-[12]. 
Chang, et al. [4] give two proposals to increase the capacity and the imperceptibility of their SVD-based schemes. The first proposal proves that the modification of the $U$ matrix column vector provides a good visible quality compared with the modification of row vectors. The second proposal consists of modifying the $\mathrm{V}$ transposed row vector coefficient in order to guarantee a less visible degradation than modifying a column vector. The manner to use the two proposals is as follows: first, they use only the U matrix, and then they used both of $\mathrm{U}$ and $\mathrm{V}$ matrices to embed the watermark. The experimental results prove that the embedded watermark in both $\mathrm{U}$ and $\mathrm{V}$ matrices considerably increases the capacity and the imperceptibility when compared with the embedded watermark only in $U$ matrix [5]. In [6], Lai proposes an image watermarking technique based on SVD, DCT, and human visual system (HVS). The host image is partitioned into $8 \times 8$ non-overlapping blocks and then the entropy and edge entropy for each block is calculated. To select embedding watermark blocks, the obtained values of entropy and edge entropy are summed and sorted into ascending order. The 2D-DCT, then the SVD transforms were applied for each selected block. To embed the watermark, some entries of the $U$ orthogonal matrix, which are $\mathrm{U}_{31}$ and $\mathrm{U}_{41}$ of each selected block were, modified.

In the light of the works presented above, we propose in this paper a blind hybrid watermarking technique using the two-dimensional DCT (2D-DCT) and the SVD transforms.

The novelty introduced in this paper is to exploit beneficially the well-known anisotropic diffusion technique of Perona-Malik in image watermarking. This technique, which finds its main use in the field of image de-noising, consists of reducing the noise of the images. In the proposed image watermarking method, the Lorentzian function is calculated and both high gradient (edge pixels) and low gradient (region pixels) are extracted according to a chosen gradient threshold. After that, the incrustation of the watermark is done in edge pixels to achieve the compromise robustness and imperceptibility.

The watermark is a logo image with a good capacity ( $32 \times 32$ pixels). In order to provide high imperceptibility, the embedding is done in a limited number of regions of the cover image. The regions chosen to host the watermark present a relatively high edges concentration, since the human visual system is less sensitive in textured, edge, and rapid-changing regions. The regions are determined by anisotropic diffusion, also called Perona-Malik diffusion.

The host image is partitioned into $8 \times 8$ non-overlapping blocks and then the coefficient of diffusion for each block is calculated. The obtained values are sorted into ascending order in view to select the blocks for embedding watermark. The 2D-DCT, then the SVD transforms are applied for each selected block. After that the watermark is embedded by modifying the $U$ matrix components $U_{31}$ and $U_{41}$.

The proposed method can find its usefulness in many watermarking applications, such as:

- Ownership Claim, which means that a legal owner can prove his ownership by retrieving the watermark (signature) from its digital content;

- Copy Control and Fingerprinting where individual watermarks are embedded in each distributed copy of the content. This prevents buyers of the content from making illegal copies because they will be identified;

- Content Authentication permits to ensure the integrity of the watermarked image, which means that it has not been altered by trying to remove the watermark.

The proposed work is organized as follows. In Section II, an overview of the 2D-DCT and the SVD transforms is given. The Perona-Malik diffusion is outlined in Section III. In section IV, the proposed watermarking algorithm, with its insertion and extraction phases, is presented. Experimental results are discussed in Section $\mathrm{V}$ and conclusions are given in Section VI.

\section{OVERVIEW OF THE 2D-DCT AND THE SVD}

\section{A. The $2 D-D C T$}

The 2D-DCT, which is a direct extension of the 1D-DCT, is a frequently used transform in image processing [7]. It transforms a 2D signal, an image for example, from its spatial representation into its frequency representation, where most of the energy is concentrated in the lower frequencies. Actually, the JPEG image compression standards rely on this property by discarding the higher frequency coefficients since perceptually significant components normally correspond to low and mid-band frequencies. Bearing this in mind, many of the watermarking techniques that use the $2 \mathrm{D}$-DCT embed the watermark in the mid-band frequencies of the cover to gain robustness against JPEG compression.

For an image $M$ of an $(N \times N)$ dimension, the 2D-DCT and its inverse 2D-IDCT are given by the formulas, respectively:

$$
\begin{gathered}
F(u, v)=\sum_{i=0}^{N-1} \sum_{j=0}^{N-1} C(u) C(v) f(i, j) \cos \left\lfloor\frac{\pi(2 i+1) u}{2 N}\right\rfloor \times \\
\times \cos \left[\frac{\pi(2 j+1) v}{2 N}\right], \\
f(i, j)=\sum_{u=0}^{N-1} \sum_{v=0}^{N-1} C(u) C(v) F(u, v) \cos \left\lfloor\frac{\pi(2 i+1) u}{2 N}\right\rfloor \times \\
\times \cos \left[\frac{\pi(2 j+1) v}{2 N}\right],
\end{gathered}
$$

with

$$
C(u)=\left\{\begin{array}{lc}
\sqrt{\frac{1}{N}}, & u=0, \\
\sqrt{\frac{2}{N}}, & u=1,2, \ldots, N-1,
\end{array}\right.
$$

and

$$
C(v)=\left\{\begin{array}{cc}
\sqrt{\frac{1}{N}}, & v=0, \\
\sqrt{\frac{2}{N}}, & v=1,2, \ldots, N-1 .
\end{array}\right.
$$




\section{B. The SVD}

The SVD operation is one of the most useful tools of linear algebra with a number of image applications, such as image compression and watermarking [9]-[12], [19], [20]. Given a real matrix, for example an image $M$ of a $(N \times N)$ dimension, it can be decomposed into a product of three matrices specified by

$$
M=U \times S \times V^{T},
$$

where $S$ is a diagonal matrix, i.e., $S=\operatorname{diag}\left(\lambda_{1}, \lambda_{2}, \ldots, \lambda_{N}\right)$, with positive real values $\lambda_{i}$ called the singular values of $M$ satisfying $\lambda_{1}>\lambda_{2}>\ldots>\lambda_{N} . U$ and $V$ are orthogonal matrices, i.e., $U^{T} \cdot U=V^{T} \cdot V=I_{N}$. The columns of $U$ and those of $V$ are called the left-singular and the rightsingular vectors of $M$.

$M$ can also be written as

$$
\begin{gathered}
M=\lambda_{1} \times U_{1} \times V_{1}^{T}+\lambda_{2} \times U_{2} \times V_{2}^{T}+ \\
+\ldots+\lambda_{r} \times U_{r} \times V_{r}^{T},
\end{gathered}
$$

where $r$ is the rank of the matrix $M$.

When SVD is applied to an image, it is important to note that each singular value specifies the luminance of one image layer while the corresponding pair of singular vectors specifies the geometry of the image layer.

In the SVD decomposition, the $U$ matrix shows two important characteristics related to its first column elements: all these elements have the same sign and their values are very close. This is shown in the subsequent example below with a $(4 \times 4)$ matrix $M$ issued from an image [10]

$$
M=\left\lfloor\begin{array}{llll}
76 & 77 & 79 & 87 \\
82 & 86 & 86 & 95 \\
74 & 75 & 76 & 84 \\
77 & 79 & 82 & 83
\end{array}\right\rfloor
$$

After applying the SVD transform to the matrix $M$, we obtain the $U$ matrix given as follows

$$
U=\left[\begin{array}{cccc}
-0.4912 & -0.2831 & -0.4798 & -0.6696 \\
-0.5374 & -0.3098 & 0.7835 & -0.0362 \\
-0.4757 & -0.2601 & -0.3948 & 0.7418 \\
-0.4936 & 0.8696 & 0.0048 & -0.0091
\end{array}\right] \text {. }
$$

\section{THE PERONA-MALIK DIFFUSION}

The smoothness of the image is evaluated according to the quantity of abrupt variations of the spatial values of the image, which is expressed by the proportion of the contribution of the high frequencies in the energy of the image. To study this disturbance, we must find an effective means of measurement and we must also set a scale of measurement, which allows us to distinguish and compare the different levels of smoothness. In this paper, we use the diffusion-based models proposed by Perona-Malik in the construction of a diffusion operator that depending on the local properties of the image permits to create a filter that keeps seeing the outlines and blurs of the low gradient areas.

The technique of Perona-Malik anisotropic diffusion is originally used in the reduction of noise in an image without removing the significant parts of its contents, such as the edges, the lines or other important details [13]-[15].

Using the same reasoning as in image de-noising, our idea consists of embedding the watermark in convincing emplacements, by which we realize the robustness and imperceptibility compromise while conserving any image proprieties like the edges, the lines, and other important details.

\section{Mathematical formulas}

The diffusion equation presented by Pietro Perona and Jitendra Malik in [16] use a partial differential equation (PDE), which is an equation that includes the partial derivatives of an unknown function of more than one independent variable[17], [18].

Consider the anisotropic diffusion equation in the continuous domain as

$$
\left\{\begin{array}{c}
\frac{\partial u(x, y, t)}{\partial t}=\operatorname{div}(g(|\nabla u(x, y, t)|) \times \nabla u(x, y, t)), \\
u(t=0)=u_{0},
\end{array}\right.
$$

where div, $\nabla,|\cdot|, \nabla u, u_{0}$, and $g(|\nabla u|)$ are divergence operator, the gradient operator, module of the original image, and the positive and strictly decreasing diffusion coefficient, respectively that satisfy the following boundary conditions:

$$
\left\{\begin{array}{c}
g(0)=1, \\
\lim _{x \rightarrow \infty} g(\nabla u)=0 .
\end{array}\right.
$$

Perona and Malik proposed a function that satisfies the two previous conditions. It is a Lorentzian function, which is defined by the relation and is expressed as

$$
g(|\nabla u|)=\frac{1}{1+\left(\frac{|\nabla u|}{k}\right)^{2}},
$$

where $k$ is the gradient threshold, from which we decide whether the amplitude of the gradient is strong (edge pixels) or weak (region pixels) [16].

If $|\nabla u| \leq k$, then the PDE enhances the contours. The points in the gradient norm that are less than $k$ are considered noise while the others are considered to be contours. In other words, it serves to set the limit between strong gradients and low gradients.

\section{THE PROPOSED WATERMARKING ALGORITHM}

In this section, we present the proposed embedding and extraction processes, which are illustrated in Fig. 1 and Fig. 2 , respectively. The technique of Perona-Malik diffusion presented in Fig. 3, which is used to select the edges to embed the watermark, increases the robustness of the watermark against most attacks without affecting the visual 
quality of the image.

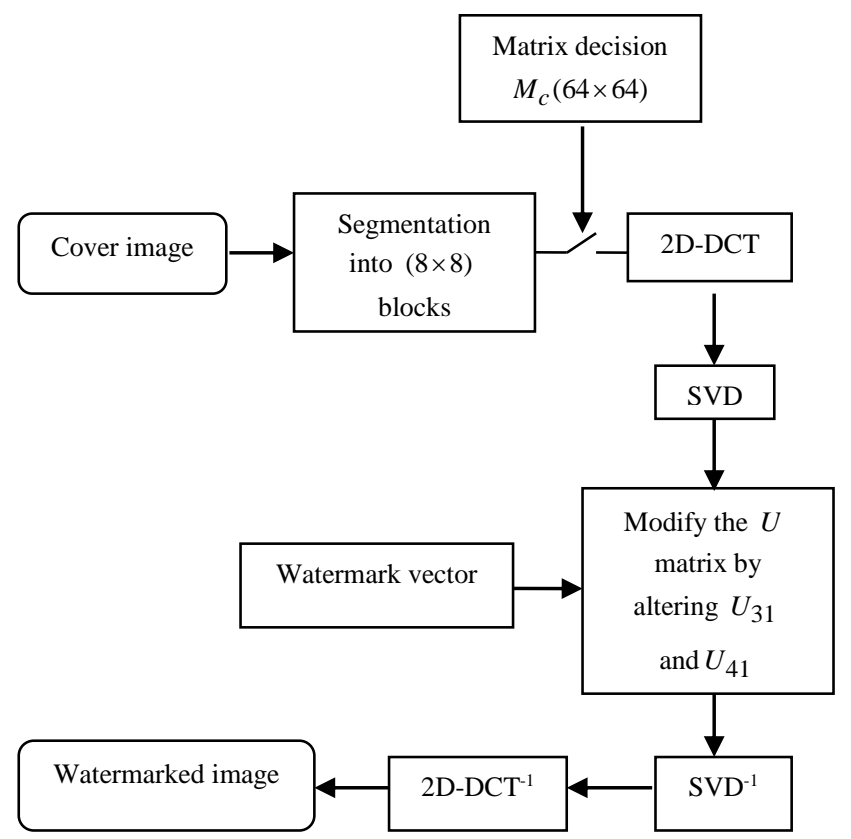

Fig. 1. Block diagram of the watermarking embedding process.

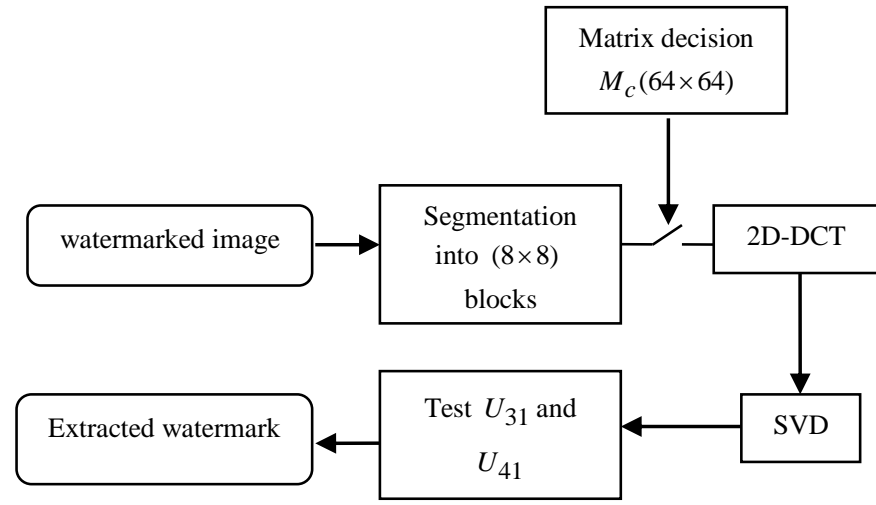

Fig. 2. Block diagram of the watermarking extraction process.

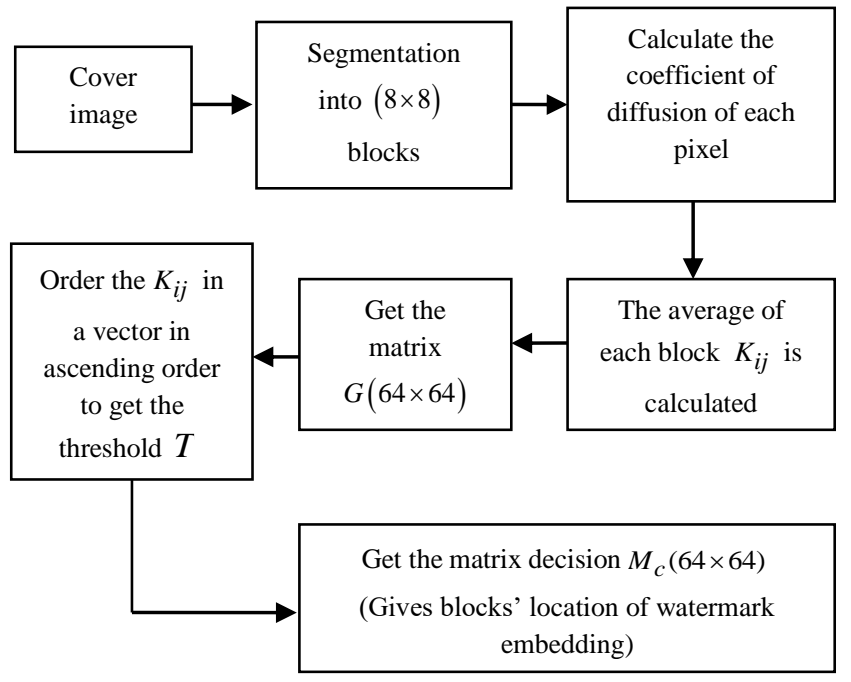

Fig. 3. Block diagram of the Perona-Malik technique.

\section{A. The Embedding Process}

The original image is of size $(m \times n)$ and the watermark image is of size $\left(m_{1} \times n_{1}\right)$. The watermark embedding procedure is as follows.

Step 01: The original image is first partitioned into non- overlapping blocks with $(8 \times 8)$ pixels and then a diffusion coefficient is calculated for each pixel of these blocks;

Step 02: An average diffusion coefficient $K_{i j}$ is calculated for each block $(i, j)$ and a matrix $G$ of size $(m \times n)$ is constructed, and filled up with all those diffusion coefficients;

Step 03: The diffusion coefficients $K_{i j}$ are ordered in a vector with the purpose of choosing a threshold $t$, which will allow us to select the appropriate blocks for watermark embedding.

Step 04: A binary matrix $M_{c}$ of size $\left(m_{1} \times n_{1}\right)$, which is the key matrix for insertion is created in the following way:

$$
\left\{\begin{array}{c}
M_{c}(i, j)=0, \quad \text { if } \quad G(i, j) \leq t, \\
M_{c}(i, j)=1, \quad \text { otherwise }
\end{array}\right.
$$

with $1 \leq i, j \leq \frac{m}{r}$.

$M_{c}$ allows us to get the watermarked blocks.

Step 05: The watermark $W$ is reshaped into a vector $w$ of length $m_{1} \times n_{1}$.

Step 06: If $M_{c}(i, j)=1$ (with $1 \leq i, j \leq \frac{m}{r}$ ), then:

Apply the 2D-DCT transform and then the SVD to the selected block $(i, j)$

Calculate:

$$
x=\frac{\left(\left|U_{31}\right|+\left|U_{41}\right|\right)}{T},
$$

where $T$ is a threshold and $U_{31}$ and $U_{41}$ are the third and fourth elements of the $U$ matrix, repectively.

Modify the $U$ matrix of each selected block as follows:

$$
\left\{\begin{array}{l}
U_{31}=x+\frac{T}{2}, U_{41}=x-\frac{T}{2}, \text { if } w(l)=1, \\
U_{31}=x-\frac{T}{2}, U_{41}=x+\frac{T}{2}, \text { if } w(l)=0
\end{array}\right.
$$

with $1 \leq l \leq m_{1} \times n_{1}$.

Step 07: Apply the inverse SVD and then the inverse 2DDCT transforms to each selected block to obtain the watermarked image $I_{w}$.

\section{B. The Extraction Process}

Step 01: Use the key matrix $M_{c}$ to get the watermarked blocks.

Step 02: Apply the 2D-DCT and then the SVD to the watermarked blocks.

Step 03: The watermark is extracted as a vector $w^{\prime}$ using the following equation:

$$
\left\{\begin{array}{l}
w^{\prime}=0, \quad \text { if } \quad U_{31}^{\prime} \geq U_{41}^{\prime}, \\
w^{\prime}=1, \quad \text { if } \quad U_{31}^{\prime} \leq U_{41}^{\prime},
\end{array}\right.
$$

where $U_{31}^{\prime}$ and $U_{41}^{\prime}$ are the third and fourth elements of the 
first column of the $U^{\prime}$ matrix, respectively.

Step 04: The final extracted watermark $W^{\prime}$ is obtained by transforming $w$ ' vector into a matrix.

\section{EXPERIMENTAL RESULTS}

In this section, the results of some experiments carried out

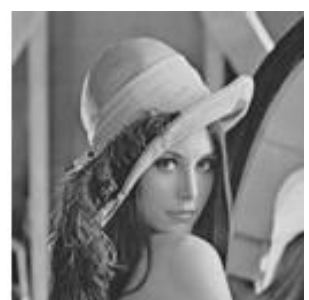

(a)

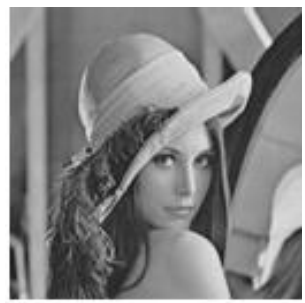

(e)

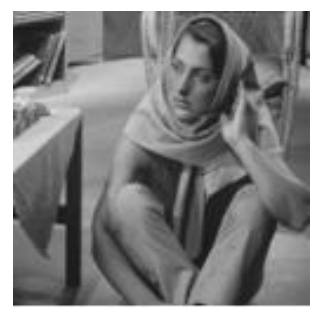

(b)

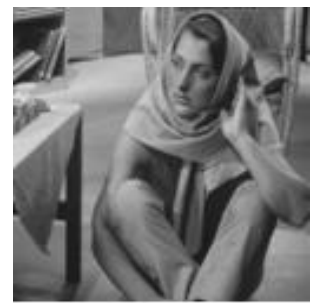

(f)

in order to evaluate the performance of the proposed watermarking scheme by using MATLAB are discussed. Four standard gray-level images of $(512 \times 512)$ pixels, which are Lena, Baboon, Barbara, and Goldhill, are used as cover images. The watermark of good capacity is a $(32 \times 32)$ binary image. The cover and the watermarked images are illustrated in Fig. 4.

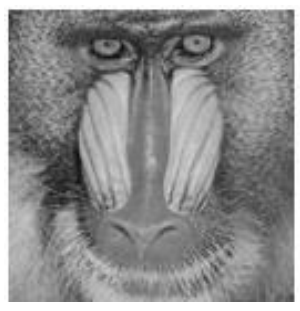

(c)

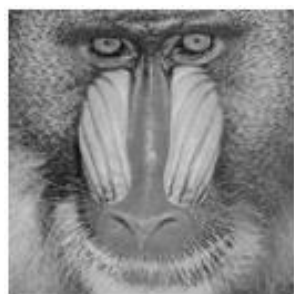

(g)

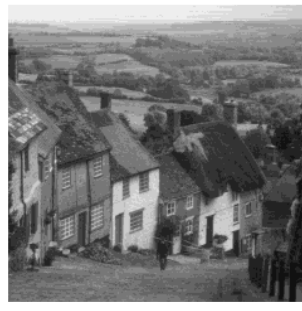

(d)

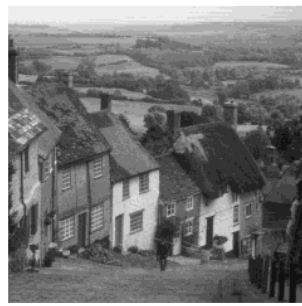

(h)

Fig. 4. Original images: (a) Lena; (b) Barbara, (c) Baboon, and (d) Goldhill; watermarked images: (e) Lena, (f) Barbara, (g) Baboon, and (h) Goldhill.

In order to evaluate the quality of the watermarked images in terms of watermark imperceptibility in our proposed method, we use the peak signal-to-noise ratio (PSNR) given in (14) [19] as:

$$
\begin{gathered}
P S N R=10 \log _{10}\left(\frac{255^{2}}{M S E}\right), \\
M S E=\frac{1}{m \times n} \sum_{i=1}^{m} \sum_{j=1}^{n}[x(i, j)-y(i, j)]^{2},
\end{gathered}
$$

where $m, n$ are the image size, $x(i, j)$ is the pixel value in the original image, and $y(i, j)$ is the pixel value in the watermarked image.

The degree of similarity between the incrusted watermark and the extracted one is evaluated by using the bit correction rate (BCR) given by [19]

$$
B C R=\frac{\sum_{i=1}^{M} \sum_{j=1}^{N} \overline{W_{i j} \otimes W_{i j}^{\prime}}}{M \times N},
$$

where $\otimes$ is the OR exclusive operator, $(M, N)$ are the size of the watermark, $W$ is the binary value of the incrusted watermark, and $W^{\prime}$ is the binary value of the extracted one.

Greater BCR corresponds to better robustness.

Table I summarizes the PSNR and BCR results obtained for different test images. Except for Baboon image, the PSNR values show high imperceptibility of the proposed technique. The BCR values are equal to 1 for Lena, Barbara, and Gold hill images. It is very close to 1 for Baboon image. TABLE I. PSNR AND BCR VALUES FOR THE HOST IMAGES LENA, BABOON, BARBARA AND GOLD HILL WITH THRESHOLD T $=0.02$.

\begin{tabular}{|c|c|c|}
\hline & PSNR & BCR \\
\hline Lena & 46.1090 & 1.00000 \\
\hline Baboon & 34.7189 & 0.99990 \\
\hline Barbara & 42.9475 & 1.00000 \\
\hline Gold hill & 38.6053 & 1.00000 \\
\hline
\end{tabular}

Since the image Baboon is strongly textured and presents high frequency components when extracting the watermark, slight rounding errors may occur. This affects the BCR calculation. Nevertheless, this error is minimal (of the order of 0.0001) and does not affect the obtained results. These results prove that the extracted watermark is very similar to the incrusted one.

Table II presents the PSNR and BCR results using four different threshold $T$ values: $0.002,0.012,0.02$, and 0.04 . These results show that the greater the threshold $T$, the lower the PSNR and the higher the BCR. This means that a high threshold $T$ provides a low imperceptibility, but a high robustness and vice versa.

TABLE II. PSNR AND BCR VALUES FOR THE HOST IMAGE LENA UNDER DIFFERENT THRESHOLD VALUES.

\begin{tabular}{|c|c|c|c|c|}
\hline Threshold & $\mathbf{0 . 0 0 2}$ & $\mathbf{0 . 0 1 2}$ & $\mathbf{0 . 0 2}$ & $\mathbf{0 . 0 4}$ \\
\hline PSNR & 54.9890 & 49.6954 & 46.1090 & 40.4730 \\
\hline BCR & 0.9639 & 1.00000 & 1.00000 & 1.00000 \\
\hline
\end{tabular}

In Table III, a comparison of PSNR values related to Lena's image is done between our method and the previous ones [4], [5], [12], and [6] for different threshold values without attacks. When taking into account the average values of Table III, our results are slightly superior to the results of [4], [5], and [12] except the ones of [6]. However, 
when looking at the results provided with thresholds superior to 0.002 ours outperform those of [6].

TABLE III. PSNR PERFORMANCE FOR LENA IMAGE UNDER DIFFERENT THRESHOLD VALUES

\begin{tabular}{|c|c|c|c|c|c|}
\hline Threshold & $\begin{array}{c}\text { Chang, } \\
\text { et al [4] }\end{array}$ & $\begin{array}{c}\text { Chung, } \\
\text { et al [5] }\end{array}$ & $\begin{array}{c}\text { Fan, et } \\
\text { al [12] }\end{array}$ & Lai [6] & Ours \\
\hline 0.002 & 48.80 & 50.17 & 48.91 & 61.69 & 54.98 \\
\hline 0.012 & 48.02 & 47.83 & 48.12 & 49.37 & 49.65 \\
\hline 0.02 & 46.90 & 45.94 & 46.98 & 44.75 & 46.10 \\
\hline 0.04 & 43.74 & 42.04 & 43.81 & 38.51 & 40.47 \\
\hline Average & 46.865 & 46.495 & 46.955 & 48.58 & 47.80 \\
\hline
\end{tabular}

In order to evaluate the robustness of our proposed method, several attacks are applied to the watermarked image (Table IV): JPEG compression, noise addition (Gaussian noise, salt and pepper, and speckle noise), denoising attack (Median filter attack and Sharpening attack), and cropping. These attacks are also extended to Baboon and Barbara watermarked images as shown in Table V. The results obtained are very satisfactory, especially for compression and filtering tests.

TABLE IV. BCR VALUES FOR LENA IMAGE UNDER DIFFERENT ATTACKS WITH THRESHOLD $T=0.02$.

\begin{tabular}{|c|c|}
\hline Attacks & BCR \\
\hline JPEG compression $70 \%$ & 0.9600 \\
\hline Gaussian noise $1 \%$ & 0.7627 \\
\hline Salt and pepper1\% & 0.9131 \\
\hline Speckle noise $4 \%$ & 0.7275 \\
\hline Median filter $3 \times 3$ & 1.0000 \\
\hline Sharpening & 1.0000 \\
\hline Upper right Cropping & 0.8506 \\
\hline
\end{tabular}

TABLE V. BCR PERFORMANCE UNDER DIFFERENT ATTACKS WITH THRESHOLD $T=0.04$.

\begin{tabular}{|c|c|c|c|c|c|}
\hline Attacks & $\begin{array}{c}\text { Chang, } \\
\text { et al. } \\
{[\mathbf{4}]}\end{array}$ & $\begin{array}{c}\text { Chung, } \\
\text { et al. }[\mathbf{5}]\end{array}$ & $\begin{array}{c}\text { Fan, } \text { et } \\
\text { al. } \\
{[\mathbf{1 2}]}\end{array}$ & $\begin{array}{c}\text { Lai } \\
{[\mathbf{6}]}\end{array}$ & Ours \\
\hline $\begin{array}{c}\text { Upper left } \\
\text { CR }\end{array}$ & 0.8125 & 0.8125 & 0.8125 & 0.8477 & 0.8506 \\
\hline GN 1\% & 0.8750 & 0.9179 & 0.8740 & 0.9521 & 0.9199 \\
\hline MF 3 3 & 0.5507 & 0.5107 & 0.5468 & 1.0000 & 1.0000 \\
\hline JPEG70\% & 0.9687 & 0.9794 & 0.9658 & 1.0000 & 1.0000 \\
\hline SH & 0.8847 & 0.9892 & 0.9873 & 1.0000 & 1.0000 \\
\hline
\end{tabular}

A comparison, in terms of BCR values between our method and techniques presented in [4], [5], [12], and [6] under various attacks on Lena's image is given in Table $\mathrm{V}$ and Table VI (CR: cropping, GN: Gaussian noise, MF: median filter, JPEG compression, and SH: sharpening).

TABLE VI. BCR PERFORMANCE UNDER DIFFERENT ATTACKS WITH THRESHOLD $T=0.012$.

\begin{tabular}{|c|c|c|c|c|c|}
\hline Attacks & $\begin{array}{c}\text { Chang, } \\
\text { et al. } \text { [4] }\end{array}$ & $\begin{array}{c}\text { Chung, } \\
\text { et al [5] }\end{array}$ & $\begin{array}{c}\text { Fan, } \text { et } \\
\text { al. } \text { [12] }\end{array}$ & $\begin{array}{c}\text { Lai } \\
{[\text { [6] }}\end{array}$ & Ours \\
\hline $\begin{array}{c}\text { Upper left } \\
\text { CR }\end{array}$ & 0.8115 & 0.8115 & 0.8115 & 0.8476 & 0.8506 \\
\hline GN 1\% & 0.6230 & 0.7080 & 0.6250 & 0.5928 & 0.6680 \\
\hline MF 3 3 3 & 0.5283 & 0.4980 & 0.5292 & 0.9688 & 0.9980 \\
\hline JPEG70\% & 0.6806 & 0.6855 & 0.6806 & 0.9941 & 0.9463 \\
\hline SH & 0.7056 & 0.7323 & 0.7066 & 0.8805 & 1.0000 \\
\hline
\end{tabular}

The results presented in Table V and Table VI show that the proposed method outperforms the cited methods in terms of robustness and imperceptibility whereas [6] is a little better than our method in term of BCR. This confirms the efficiency of the proposed method.

\section{CONCLUSIONS}

In this paper, a hybrid image watermarking technique based on DCT-SVD and human visual characteristics is presented. This technique contributes to the protection of digital images by fully exploiting the relevant characteristics of SVD, which efficiently characterizes fundamental algebraic properties of an image conjointly with the DCT transform used essentially in the JPEG image compression. The use of HVS characteristics, provided by the PeronaMalik diffusion approach, helps to select watermark embedding blocks for a good compromise between robustness $(0.9639 \leq \mathrm{BCR} \leq 1.00000)$ and imperceptibility $(40.4730 \leq$ PSNR $\leq 54.9890)$ of the watermark embedded in Lena test image for different threshold values (Table A-I). Experimental results of the proposed technique have shown its efficiency when compared with existing works in terms of imperceptibility and robustness, especially against different attacks: JPEG compression, adding noise, filtering, and cropping (Table $\mathrm{V}$ and Table VI). A perspective for future works is to implement our method in digital video watermarking since a video can be decomposed to a set of consecutive digital images.

\section{APPENDIX A}

TABLE A-I. BCR PERFORMANCE VISUAL QUALITY OF THE EXTRACTED WATERMARK AND BCR VALUES UNDER DIFFERENT ATTACKS FOR LENA, BABOON, AND BARBARA

\begin{tabular}{|c|c|c|c|}
\hline \multicolumn{4}{|c|}{ IMAGES. } \\
\hline Attacks & Lena & Baboon & Barbara \\
\hline $\begin{array}{l}\text { Without } \\
\text { attacks }\end{array}$ & $\begin{array}{c}\boldsymbol{A} \\
{ }_{\mathrm{BCR}}=1.0000\end{array}$ & $\begin{array}{c}\mathbf{A} \\
\mathrm{BCR}=0.9990\end{array}$ & $\mathrm{BCR}=1.0000$ \\
\hline $\begin{array}{c}\text { Compression } \\
75 \%\end{array}$ & 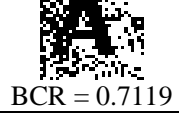 & $\begin{array}{c}\mathrm{BCR}=0.9287 \\
=0 .\end{array}$ & 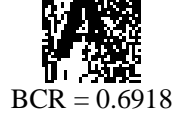 \\
\hline $\begin{array}{l}\text { Upper left } \\
\text { Cropping }\end{array}$ & $\begin{array}{c}\text { in } \\
\mathrm{BCR}=0.8506\end{array}$ & ${ }_{\mathrm{BCR}}=0.8652$ & $\mathrm{BCR}=$ \\
\hline $\begin{array}{l}\text { Lower left } \\
\text { Cropping }\end{array}$ & $\mathrm{BCR}$ & BCR= & $\mathrm{BCR}=$ \\
\hline $\begin{array}{l}\text { Upper right } \\
\text { Cropping }\end{array}$ & $\operatorname{rix}_{\mathrm{BCR}}=$ & BCR $=0.8535$ & BCR $=0.9082$ \\
\hline $\begin{array}{l}\text { Lower right } \\
\text { Cropping }\end{array}$ & $\mathrm{BCR}$ & $\begin{array}{c}\text { BCR }=0.7686 \\
\text { lith }\end{array}$ & $\begin{array}{c}\text { BCR }=0.8057 \\
\mathrm{~B}\end{array}$ \\
\hline $\begin{array}{l}\text { Salt \& pepper } \\
\quad 0.005\end{array}$ & $\begin{array}{c}\mathrm{B}= \\
\mathrm{BCR}=0.9463\end{array}$ & $\begin{array}{c}\mathrm{BCR}=0.9521 \\
\mathrm{~B}\end{array}$ & $\begin{array}{c}\boldsymbol{A} \\
\mathrm{BCR}=0.9277\end{array}$ \\
\hline $\begin{array}{c}\text { Salt \& pepper } \\
0.01\end{array}$ & $\begin{array}{c}\therefore \\
\mathrm{BCR}=0.9160\end{array}$ & $\begin{array}{c}\text { BCR }=0.9189 \\
0\end{array}$ & $\begin{array}{c}x \\
\text { BCR }=0.8750\end{array}$ \\
\hline $\begin{array}{l}\text { Salt \& pepper } \\
\quad 0.05\end{array}$ & 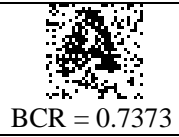 & 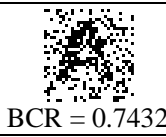 & 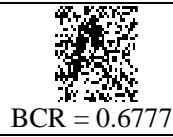 \\
\hline
\end{tabular}




\begin{tabular}{|c|c|c|c|}
\hline Attacks & Lena & Baboon & Barbara \\
\hline $\begin{array}{c}\text { Gaussian } \\
\text { noise }\end{array}$ & $\begin{array}{c}3 \mathrm{~A} \\
\text { BCR }=0.7588\end{array}$ & $\begin{array}{c} \\
\mathrm{BCR}=0.7334 \\
3_{4}\end{array}$ & 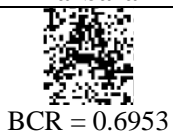 \\
\hline Speckle noise & $\begin{array}{c}46 \\
\text { BCR }=0.7080 \\
\text { कon }\end{array}$ & 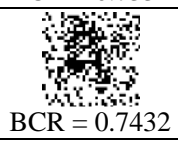 & 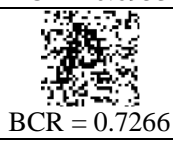 \\
\hline Median filter & $\mathrm{BCR}=1$ & $\begin{array}{c}\text { BCR }=0.9814 \\
\end{array}$ & $\begin{array}{c}\mathrm{BCR}=0.9932 \\
\mathrm{~B}\end{array}$ \\
\hline Sharpening & $\mathrm{BCR}=1$ & $\begin{array}{c}\text { BCR }=0.9805 \\
\text { (1) }\end{array}$ & $\mathrm{BCR}=0.9971$ \\
\hline
\end{tabular}

\section{REFERENCES}

[1] V. Potdar, S. Han, and E. Chang, "A survey of digital image watermarking techniques", in Proc. 3rd IEEE International Conference on Industrial Informatics (INDIN), pp 709-716, 2005 DOI: 10.1109/INDIN.2005.1560462.

[2] N. Nikolaidis and I. Pitas, "Robust image watermarking in the spatial domain”, Signal Processing, vol. 66, no. 3, pp. 385-403, 1998. DOI: 10.1016/S0165-1684(98)00017-6.

[3] T. Pardhu and B. R. Perli, "Digital image watermarking in frequency domain", in Proc. International Conference on Communication and Signal Processing (ICCSP), pp. 208-211, 2016. DOI: 10.1109/ICCSP.2016. 7754123

[4] C.-C. Chang, P. Tsai, and C.-C. Lin, "SVD-based digital image watermarking scheme", Pattern Recognition Letters, vol. 26, no. 10, pp. 1577-1586, 2005. DOI: 10.1016/j.patrec.2005.01.004.

[5] K.-L. Chung, W.-N. Yang, Y.-H. Huang, S.-T Wu, and Y.-C. Hsu, "On SVD-based watermarking algorithm", Applied Mathematics and Computation, vol. 188, no. 1, pp. 54-57, 2007. DOI: 10.1016/j.amc. 2006.09.117.

[6] C.-C. Lai, "An improved SVD-based watermarking scheme using human visual characteristics", Optics Communication, vol. 284, no. 4, pp. 938-944, 2011. DOI: 10.1016/j.optcom.2010.10.047.

[7] A.-B. Watson, "Image compression using the discrete cosine transform", Mathematica Journal, vol. 4, no. 1, pp. 81-88, 1994 DOI: 10.1007/978-3-322-96658-2_5.

[8] X. B. Kang, F. Zhao, G. F. Lin, and Y. J. Chen, "A novel hybrid of
DCT and SVD in DWT domain for robust and invisible blind image watermarking with optimal embedding strength“, Multimedia Tools and Applications, vol. 77, pp. 13197-13224, 2018. DOI: 10.1007/ c11042-018-6036-Z.

[9] L. Cao, "Singular value decomposition applied to digital image processing", Division of Computing Studies, Arizona Arizona State, University Polytechnic Campus, Mesa, Arizona 85212, pp. 1-15.

[10] C.-C. Lai, "A digital watermarking scheme based on singular value decomposition and tiny genetic algorithm", Digital Signal Processing, vol. 21, no. 4, pp. 522-527, 2011. DOI: 10.1016/j.dsp.2011.01.017.

[11] I. Nouioua, N. Amardjia, and S. Belilita, "A novel blind and robust video watermarking technique in fast motion frames based on SVD and MR-SVD', Security and Communication Networks, pp. 17, 2018. DOI: $10.1155 / 2018 / 6712065$.

[12] M.-Q. Fan, H.-X. Wang, and S.-K. Li 'Restudy on SVD-based watermarking scheme", Applied Mathematics and Computation, vol. 203, no 2, pp. 926-930, 2008. DOI: 10.1016/j.amc.2008.05.003.

[13] S.-1. Jia, "A novel blind color images watermarking based on SVD", Optik - Int. Light Electron Opt, vol. 125, no. 12, pp. 2868-2874, 2014. DOI: 10.1016/j.ijleo.2014.01.002.

[14] J. Weickert, Anisotropic diffusion on image processing. ECMI Series, B. G. Teubner, Stuttgart, 1998.

[15] A. Atlas, F. Karami, and D. Meskine, "The Perona-Malik inequality and application to image de-noising", Nonlinear analysis: Real World Applications, vol. 18, pp. 57-68, 2014. DOI: 10.1016/j.nonrwa.2013.11.006.

[16] V. Kamalaveni, R.-A. Rajalakshmi, and K.-A. Narayanankutty, "Image de-noising using variations of Perona-Malik model with different edge stopping functions", Procedia Computer Science, vol. 58, pp. 673-682, 2015, DOI: 10.1016/j.procs.2015.08.087.

[17] P. Perona and J. Malik, "Scale-space and edge detection using anisotropic diffusion", IEEE Transactions on Pattern Analysis and Machine Intelligence, vol.12, no. 7, pp. 629-639, 1990. DOI: $10.1109 / 34.56205$

[18] L. C. Evans, "Partial Differential Equations", Graduate Studies in Mathematics, vol. 19, American Mathematical Society, Providence, Rhode Island, 1998. DOI: 10.1090/gsm/019.

[19] M. B. Halima, W. Boussella, M. Charfi, and A. Alimi, " Restauration des images couleurs de documents arabes anciens basé sur les EDPs," in Laurence Likforeman-Sulemroc, pp. 103-108, 2006.

[20] N. M. Makbol, B. E. Khoo, and T. H. Rassem, "Block-based discrete wavelet transform-Singular value decomposition image watermarking scheme using human visual system characteristics", IET Image processing, vol. 10 , no. 1 , pp. $34-52$, 2015. DOI: 10.1049/ietipr.2014.0965. 